\title{
Measurement of Uranium Concentration in Some Soil Samples in Tuwaitha site in Baghdad using CR-39 Detector
}

\author{
Nidhala . H.Kadhim and Saja Salam Kadhim* \\ Department of Physics, College of Science for Women, University of Baghdad, Iraq \\ Received 01 Nov 2017, Accepted 31 Dec 2017, Available online 02 Jan 2018, Vol.8, No.1 (Jan/Feb 2018)
}

\begin{abstract}
The research aims to measure the uranium concentration of soil samples collected from different regions are located around the nuclear research center at Tuwaitha to a contamination ratio statement of these regions, which include agricultural residential regions as well as being the regions for livestock of all kinds. The nuclear track detector (CR39) was used as a method to detect uranium of through calculations depended on comparison with standard samples. Results indicate that the uranium concentration ranged between (0.4ppm) and (2.538 ppm), these results are within allowed limit that equals (11.7 ppm).
\end{abstract}

Keywords: Soil, Uranium Concentration, Contamination, CR-39 Detector, Tuwaitha, SSNTD.

\section{Introduction}

Natural radiation has always been part of the human environment. Its main components are cosmic and cosmogenic radiation, terrestrial gamma radiation from natural radionuclides in rocks and soil, and natural radioactive substances in our diet and in the air we breathe (F.Bochicchio et al, 1995). Radionuclides are found in the environment as naturally occurring elements and as products or byproducts of nuclear technologies, one of the most common radionuclides is uranium (U), all isotopes of uranium are radioactive ,so it is very important their quantity to be under control (P.Todorov et al, 2006). Uranium is a radioactive and chemical element, represents by (U) symbol, and it is a heavy metal with a very high density (18.95 g /cm3, 1.7 times higher than lead's density of 11.35 $\mathrm{g} / \mathrm{cm}^{3}$ ). Metallic uranium has a high melting point $\left(1132{ }^{\circ} \mathrm{C}\right)$ and boiling point $\left(4131{ }^{\circ} \mathrm{C}\right)$, has a tensile strength similar to most steels and it is chemically very reactive (R.L.Fleischer et al, 1975). Natural uranium consists of three isotopes. Their concentrations by mass are U238 99.276\%, U235 $0.718 \%$ and U234 0.0056\% (M.A.Misdaq et al, 2006 ; R. E.White, 2013). Recently many attempts have been made to develop the alpha sensitive plastic film (ASPF) family of the solid state nuclear detector (SSNTDs) for this purpose (H.Taskin et al,2009). CR39 is one of the solid state nuclear detectors which can response to alpha particle with high efficiency (A.Makhijani et al, 2004).

*Corresponding author's ORCID ID: 0000-0003-2992-6942; Dr. Nidhala. H.Kadhim is working as Assistant Professor DOI: https://doi.org/10.14741/ijcet.v8i01.10880
Nuclear track detector is one of the most popular detectors used to study the nature of damage product by heavily ionization radiation such as alpha particle or fission fragment, the technique of measuring the number of particle by observing their track in certain organic or inorganic materials has been used for the study of phenomena in such diverse fields as geology, astrophysics, and nuclear physics. The technique based on the damage created in a solid along the path of heavily ionizing particle (M.Gavrilescu et al, 2009) as it is a very simple technique, it can be implemented easily in field of studies, since it does not require electronic system (H.Bem et al, 2004).

\section{A. Solid State Nuclear Track Detectors (SSNTDs)}

Tracks detectors are electrically insulating solids; the resistances of these reagents ranges from 1020 to106ohms. When heavy charged particles (protons, alpha particles and fission fragments) pass through these tracks, narrow paths are formed in the form of thin traces called latent tracks. These effects indicate the amount of damage generated by detector which determines the type and energy of the falling particle (A.A.Mascarenhas et al, 2006). Nuclear tracks detectors are divided into two main sections: Organic reagents and inorganic reagents.

\section{B. (CR-39) Detector}

It is an organic nuclear tracks detector; it was invented in 1978 by (Gartwright, shirk \& Price) at the University of California, USA. Its molecular formula $\left(\mathrm{C}_{12} \mathrm{H}_{18} \mathrm{O}_{7}\right)$ 
Symbolized by CR-39 according to (Columbia Resin).The general structure of the monomer contains two groups of alleles. This enables the CR-39 monomer to polymerize and bind in a homogeneous manner to (homo polymers). This detector is characterized by a density of (1.32 g.cm-3) and has an ionization voltage of (70.2eV) and is not soluble in chemical solvents CR-39 is a polycarbonate plastic comprising poly-allyl diglycol carbonate (PADC) (K.H.AL-Ubaidi et al, 2015). The chemical form of CR-39 is $\mathrm{C} 12 \mathrm{H} 18$ 07. This plastic detector is made by polymerization of the oxydi-2, 1ethanediyl, di-2-propenyl ester of carbonic acid (K.H.Al-Ubaidi et al,2006). It contains two of monomer ally functional groups $[\mathbf{C H 2}=\mathbf{C H}-\mathbf{C H 2}-]$ and has the following structure:

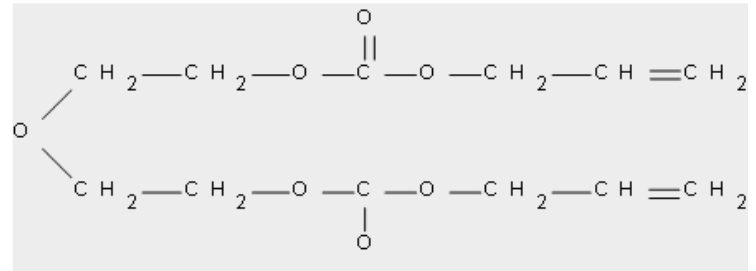

Fig.1 CR-39 structure (M.A.Al-Baidhani et al, 2006).

The CR-39 has a high sensitivity and ability to analyze the tracks of charged particles because it contains weak carbon bonds that break easily when exposed to radiation.

The general characteristics of CR-39 can be summarized as (N.F.Tawfiq et al, 2015):

1. Amorphous polymer.

2. Optically clear.

3. Environmentally very stable.

4. Having a closed packed and uniforms molecular structure.

5. Having non - solvent chemical etchant.

6. Highly cross - linked thermoset.

7. Sensitive to heavy ion damage $Z / \beta>10$ where $\beta$ is the ratio of particle velocity to the velocity of light.

\section{Radiation in Soil}

Soil is the upper part of the earth's crust and is formed as a result of rock deformation by complex physicochemical processes, which include weathering, decomposition and water movement, so the soil is the result of the action of weather and human activities on the crust rocks of the earth. The soil is naturally radioactive, because of the mineral content. The natural radioactivity may vary considerably from one type of soil to another (UNSCEAR, 1993).

Soil radionuclide activity concentration is one of the main determinants of the natural background radiation. Volcanic geographic structures as well as rocks that are rich in phosphate, granite and salt contain natural radionuclides like uranium-238, thorium-232 and potassium-40. When rocks are disintegrated through natural processes, radionuclides are carried in soil by rain and flows. In addition to the natural sources, soil radioactivity is also affected from man-made activities (Singh S. et al, 2001).

\section{Methods and material}

15 samples of soil surface samples were taken from different locations around the Tuwaitha nuclear research center in Iraq. All the samples were dried by being exposed to the sun and humidity condition for 2 days. After that these samples were placed in an electrical oven at a temperature of $80{ }^{\circ} \mathrm{C}$ for 2 hours; a special attention was paid to the samples that have been collected after the rain, thus ensuring complete removal of any residual moisture. The dried samples are crushed into a downy powder and passed through a standard $75 \mu \mathrm{m}$ mesh size. The soil and plants samples are placed in a plastic cup with plastic cover the diameter which is equal $6 \mathrm{~cm}$ and the height of the cup is $11 \mathrm{~cm}$. The cup was filled with $40 \mathrm{mg}$ of each sample. The CR-39 detector is fixed inside the cover facing the soil or plant sample and the other one was put in the bottom of the cup under the soil or plant sample. These samples were stored at about 30 days for the detection of $\alpha$-particles. The chemical etching process to the CR-39 detectors has been done in order to show the fission tracks. This operation is for the use of sodium hydroxide solution $\mathrm{NaOH}$ as an etchant solution, with normality $(6.25 \mathrm{~N})$ and temperature 80 $\mathrm{C}^{\circ}$ for ( 4 hours) as the most favorable conditions. After the etching time (4 hours), the detectors have been removed from the etchant solution by forceps and washed with distilled water and then dried.

The number of tracks on the area was determined to obtain the intensity of the tracks figures; the track density was calculated by using the following equation: $\rho=\frac{N_{\text {ave }}}{A}$

$\rho$ : Track intensity in unit: Track $/ \mathrm{mm}^{2}$

$\mathrm{N}_{\text {ave }}$ : The total tracks rate within area (A) in unit (Track)

A: the area in units $\left(\mathrm{mm}^{2}\right)$

\section{A. Uranium concentration}

Fission track technique was used for determination uranium concentration in the soil samples by making a comparison between track densities registered on the detectors of the sample and that of the standard geological sample. The uranium content in the unknown samples was determined by using the formula (A.K.Brindha et al, 2013) ; $\mathbf{C}_{\times}$(sample)/ $\mathbf{C}_{\mathbf{s}}$ (standard) $=\rho_{\times}($sample $) / \rho_{s}$ (standard) $\mathrm{C}_{\mathrm{s}}$ (standard)/ $\rho_{\mathrm{s}}$ (standard) =slope

Where:

$\mathrm{C}_{\mathrm{X}}$ : Uranium concentration in unknown sample (ppm). $\mathrm{C}_{\mathrm{s}}$ : Uranium concentration in standard sample (ppm). 
$\rho_{\mathrm{x}}$ : Track density of unknown sample(tracks $/ \mathrm{mm}^{2}$ ). $\rho_{\mathrm{s}}$ : Track density of standard sample(tracks $/ \mathrm{mm}^{2}$ ).

Fig. (2) Shows this relation, when (slope $=\rho s / C s)$

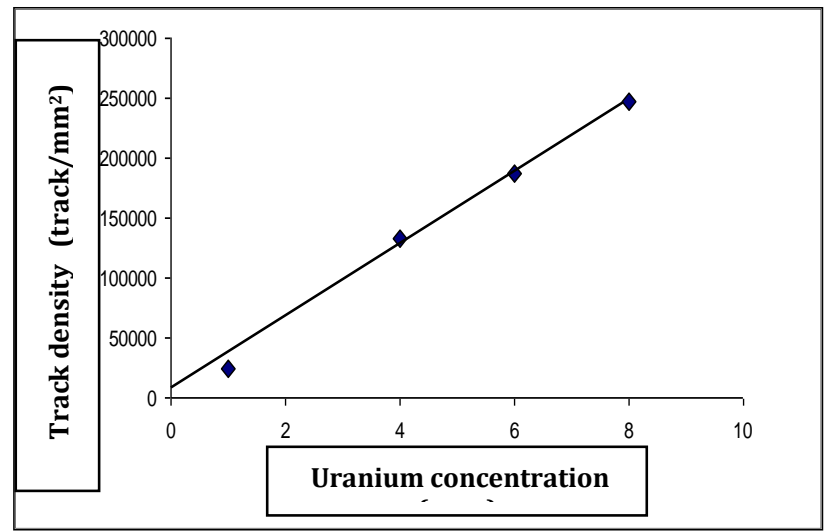

Fig.2 The relation between track density and uranium concentration for standard geological soil samples (Henryk B et al, 2004).

\section{Result and discussion}

In this research, the soil samples were used from surrounding regions of the nuclear research center at Tuwaitha to know the extent of contamination of these regions with uranium, uranium concentration has been calculated by using technique of counting the tracks of nuclear fission fragments using nuclear track detector (CR-39), and the results were arranged in Table (1) The selection of these regions to measure the ratio of uranium, depending on several factors, the most important are:

1) Because this regions are surrounding the Tuwaitha nuclear research center, which contained several nuclear reactors.

2) Because of the nuclear research center at Tuwaitha was looted by some population during the war in Iraq.

Table 1 Results of the uranium concentration in the soil samples

\begin{tabular}{ccc}
\hline $\begin{array}{c}\text { No. of } \\
\text { samples }\end{array}$ & $\begin{array}{c}\text { tracks Density } \\
\text { (tracks } / \mathbf{m m}^{2} \text { ) }\end{array}$ & $\begin{array}{c}\text { Uranium } \\
\text { concentration (ppm) }\end{array}$ \\
\hline S1 & 17314.221 & 0.57611339 \\
S2 & 50391.84 & 1.676738084 \\
S3 & 12319.75 & 0.409927362 \\
S4 & 29363.39 & 0.977037439 \\
S5 & 16193.52 & 0.538823184 \\
S6 & 28320.76 & 0.942344968 \\
S7 & 73285.14 & 2.438489748 \\
S8 & 42689.96 & 1.420465729 \\
S9 & 12821.57 & 0.42662492 \\
S10 & 15961.89 & 0.531115928 \\
S11 & 19651.32 & 0.653878022 \\
S12 & 25651.45 & 0.853526347 \\
S13 & 26651.12 & 0.886789367 \\
S14 & 76285.05 & 2.538308754 \\
S15 & 29341.77 & 0.976318055 \\
\hline
\end{tabular}

The values of uranium concentrations in soil are found to range from $\boldsymbol{S}_{\mathbf{3}} 0.4 \mathrm{ppm}$ to $\boldsymbol{S}_{\mathbf{1 4}} 2.538 \mathrm{ppm}$ Measurements of uranium concentrations in soil are important from the health protection point of view, so simple and reliable analytical methods must be available.

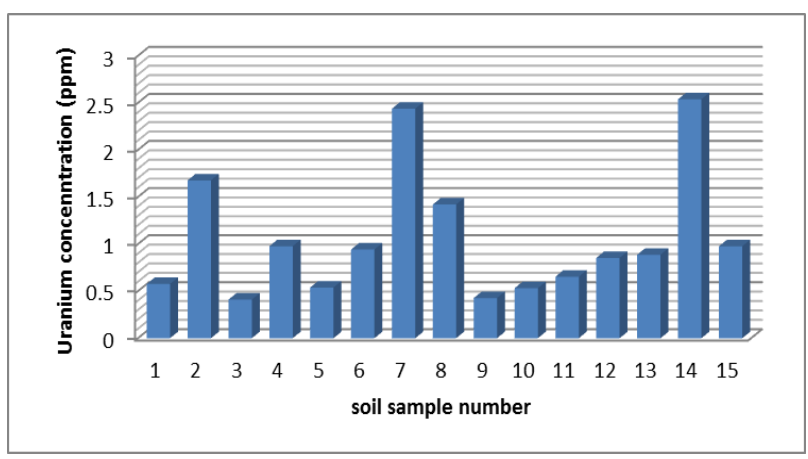

Figure 3 The concentration of uranium in the soil samples

Therefore, it was necessary to monitor the level of pollution in those regions for estimating the dangers arising from pollution and processed as quickly and the best ways to ensure the safety of the population. Through the table (1) and Figure (3).

\section{Conclusion}

1) The highest concentration of uranium in the soil samples was in the sample (S5) which is equal to ( $2.538308754 \mathrm{ppm})$, this value is less than the allowed limit.

2) The pollution ratio in the region inside AL-Tuwaitha N.R.C.) with uranium is the highest in the comparison with other regions, and this means that the people of this region are the most vulnerable to uranium from the other regions.

3) The uranium contamination ratio in region near ALTuwaitha N.R.C.) and the region (Near a large mound of dirt AL-Tuwaitha N.R.C.) despite being within allowed limit, but it is the ratio cannot be underestimated, the uranium ratio is relatively high, so it is advisable to processed with all means to ensure the safety of the population from continuous exposure to uranium, while the rest of the proportions of the other regions are reasonable proportions.

\section{References}

F. Bochicchio, J. McLaughling and S. Piermattei, (1995), Radon in indoor air, (European Collaborative Action, Report No. 15 , P. 50)

P. Todorov and E. N. Ilieva, (2006), Contamination with uranium from natural and anthropological sources, Rom. Journ. Phys., Vol.51(1-2), (27-34).

R. L. Fleischer, P. B. Price and R.M. Walker, (1975), Nuclear Tracks in Solids, Principles and Applications. (Berkeley, USA, University of California Press).

M. A. Misdaq and H. Ouabi, (2006), 238U and 232Th concentrations in various potable waters in Morocco, J. of Rad. Analy. Nucl. Chem., Vol.270(3), (543-553). 
R. E. White, (2013), Principles and practice of soil science: the soil as a natural resource. (John Wiley \& Sons).

H. Taskin and et al., (2009), Radionuclide concentrations in soil and lifetime cancer risk due to gamma radioactivity in Kirklareli Turkey, Journal of environmental radioactivity, Vol.100(1), (49-53).

A. Makhijani, L. Chalmers and B. Smith, (2004), Uranium enrichment: Just plain facts to fuel an informed debate on nuclear proliferation and nuclear power, Institute for Energy and Environmental Research, Vol.15.

M. Gavrilescu, L. V. Pavel and I. Cretescu , (2009), Characterization and remediation of soils contaminated with uranium, Journal of Hazardous Materials, Vol.163(2), (475-510).

H. Bem and F. Bou-Rabee, (2004), Environmental and health consequences of depleted uranium use in the 1991 Gulf War, Environment international, Vol.30(1),(123-134).

A. A. Mascarenhas and et al. , (2006), New polymers for solid state nuclear track detection, Radiation measurements, Vol.41(1), (23-30).

K. H. AL-Ubaidi, S. K. Nasri and Z. A. Saudany, (2015), Natural Radionuclides and Hazards in Water and Sediment Samples of Tigris River in Al- Amara city - Maysan - Iraq, Advances in Physics Theories and Applications, Vol.44 (117-122).
K. H. Al-Ubaidi, (2006), Identification and Measurements of Natural and Industrial Radioactive Pollutants in Environment of Baghdad City using Gamma Spectrometry and Solid State Nuclear Track Detector CR-39 ,PhD Thesis, Ibn Al-Haitham College,Baghdad University , Iraq.

M. A. Al-Baidhani, (2006), Determination of the Radioactivity in Soil and Water in Baghdad, Karbala and Basrah Samples,M. Sc Thesis, AL-Nahrain University College of Science.

N. F. Tawfiq, H. L. Mansour and M. S. Karim, (2015), Measurement of Radon Gas Concentrations in Tap Water for Baghdad Governorate by Using Nuclear Track Detector (CR-39), International Journal of Physics, Vol.3(6), (233238).

(UNSCEAR) United Nations Scientific Committee on the effect of Atomic Radiation, (1993), Sources, Effect, and Risks of Ionizing Radiation, (Report to the general Assembly with Scientific Annexes, United Nation).

Singh S., Malhotra R., Kumar J., Singh B. and Singh L. (2001) Rad Meas, Vol. 34, 427-431.

A K. Brindha L. Elango (2013), Occurrence of uranium in groundwater of a shallow granitic aquifer and its suitability for domestic use in southern India, J Radioanal Nucl Chem 295:357-367.

Henryk B., Firyal B. (2004) Environmental and Health Consequences of DU Use in the 1991 Gulf War, Environment International. 30, 123-134. 